\title{
Simulated environmental photo- and auto-oxidation of ambrein
}

\author{
Steven J. Rowland*, Dominic Payne and Paul A. Sutton
}

Petroleum \& Environmental Geochemistry Group, Biogeochemistry Research Centre, University of Plymouth, Drake Circus, Plymouth, PL4 8AA, Devon, U.K.

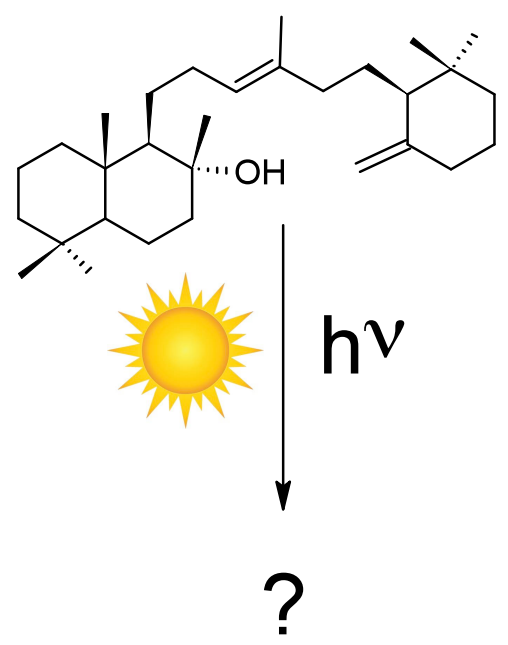

*To whom correspondence should be addressed (srowland@plymouth.ac.uk).

Paul A. Sutton (pasutton @plymouth.ac.uk) 
This paper is a non-peer reviewed preprint submitted to EarthArXiv.

\begin{abstract}
In alcoholic solutions (tinctures), the tricyclic triterpenoid alcohol, ambrein, which is usually the main ingredient of the natural product ambergris, undergoes photo-oxidation to a number of odorous products. Such tinctures are valuable in perfumery.
\end{abstract}

Similar reactions are thought to occur in the marine environment, when flotsam and jetsam ambergris are exposed to sunlight. However, previous simulations of this process used artificial photosensitisers and did not include dark controls.

We therefore conducted experiments in which ambrein in seawater $\left(\sim 1666 \mathrm{mg} \mathrm{L}^{-1}\right)$ containing a photosensitiser, forms of which are found naturally in ambergris $(5,10,15,20$ tetraphenyl-21H, $23 \mathrm{H}$-porphine copper (II); $1 \%$ of ambrein $\mathrm{w} / \mathrm{w})$, was exposed to simulated natural sunlight for experimental periods of up to $6 \mathrm{~h}$ (approximately equivalent to $48 \mathrm{~h}$ natural exposure, Florida midday, mid-summer sunlight). Dark controls were treated in the same manner, without exposure to light.

After $6 \mathrm{~h}$ exposure to light, total reaction products detectable by gas chromatography-mass spectrometry of solvent extracts, comprised about $1 \%$ by mass of the initial ambrein. However, unaccounted-for mass losses of ambrein were much greater than this $(\sim 50 \%)$, even in the dark, suggesting major losses of volatile products and/or non-recovery of some oxidation products by extraction. Photo-oxidation products included ambroxan, $\mathrm{Y}$ dihydroionone, a tricyclic enol ether and possibly a related lactone. All of these have been reported previously in photoexposures of ambrein made under, arguably, less environmentally-realistic conditions. The latter products were not observed in dark controls however, suggesting they were only products of photo-oxidation. 
This paper is a non-peer reviewed preprint submitted to EarthArXiv.

\section{Introduction}

Ambrein (I: Figure 1) is a tricyclic triterpenoid alcohol, which to date has been found naturally only in ambergris, which is a natural product of the sperm whale (reviewed by Clarke, 2006). Although ambrein is odourless, steam distillates and alcoholic solutions of ambergris (known as tinctures; Anon, 1976; Mookerjee and Patel, 1977; Awano et al., 2005), contain a number of lower molecular weight, volatile and semi-volatile, odorous products of photo- and possibly, auto-oxidation (usually <0.3\%; Ruzicka et al., 1948; Mookerjeee and Patel 1977), which are valued in perfumery.

Similar oxidation processes are thought to occur for ambergris found as flotsam or jetsam in the natural environment. Indeed, some attempts have been made to simulate this (e.g. Ohloff et al., 1977).

In their study, Ohloff et al., dissolved ambrein in toluene at a concentration of $42 \mathrm{~g} \mathrm{~L}^{-1}$, in the presence of dinaphthylenethiophene as a photosensitiser. Samples were exposed for $1.5 \mathrm{~h}$ to radiation from a high-pressure mercury lamp at $18-20^{\circ} \mathrm{C}$. Shen et al. (2007) exposed ambrein in acetone at $60 \mathrm{~g} \mathrm{~L}^{-1}$, to shortwave UV light $(254 \mathrm{~nm})$ for $72 \mathrm{~h}$ at room temperature.

Both of the above studies identified a number of oxidation products but clearly, the above conditions (very high concentrations, use of organic solvents, use of artificial sensitisers, UV irradiation), are unlikely to be representative of those found in the natural environment.

In the present, albeit preliminary study, we sought to try to better simulate the natural conditions of photo exposure of ambrein, by using a photosensitiser that occurs naturally in ambergris, by using seawater as a matrix and using radiation which simulated the spectrum of natural sunlight. We also studied dark controls to investigate auto-oxidation.

\section{Experimental}

\subsection{Ambergris sample}

Small amounts of ambergris, taken from a boulder weighing $\sim 6.3 \mathrm{~kg}$, were supplied to our laboratory.

\subsection{Isolation and characterisation of ambrein}

\subsubsection{Column chromatography}


This paper is a non-peer reviewed preprint submitted to EarthArXiv.

A piece of the ambergris was extracted and fractionated, essentially by the methods of Hardwick and Laws (1951). Thus, ca. $0.7 \mathrm{~g}$ was weighed into a pre-weighed vial and dichloromethane (DCM; $4 \mathrm{~mL}$ ) was added, followed by vortex mixing (10 s), then sonication (5 min, sweep function, $30^{\circ} \mathrm{C}$ ). A dark yellow-orange cloudy solution was observed. The liquid was decanted through a pre-extracted (Soxhlet, DCM, $8 \mathrm{~h}$ ) cotton wool plug, in a 10 $\mathrm{cm}$ burette. After filtering, the solution was less cloudy and there were small black and brown fragments left on the cotton wool plug. The solvent was removed by a gentle stream of nitrogen. The extract $(0.754 \mathrm{~g})$ was dissolved in a minimal amount of DCM and was added to a $20 \mathrm{~g}$ silica chromatography column. The tap was opened to draw the sample onto the silica, when a dark orange band was observed. Fractions $A-K$ were obtained in round bottom flasks by sequential elution using increasingly polar solvents (Table 1). The solvent in each flask was removed by rotary evaporation. A few drops of DCM were added to each flask and the fractions were transferred to pre-weighed $7 \mathrm{~mL}$ vials. The solvent was then removed from each by nitrogen blowdown $\left(40^{\circ} \mathrm{C}\right)$ until dry. The total mass of all the fractions was $0.755 \mathrm{~g}$, resulting in $\sim 100 \%$ recovery of material from the start of the chromatography separation.

\subsubsection{Melting point determination}

The melting point of Fraction E was determined using a Leica VMHB system Kofler block, (Type 184306 series No. 418058,) calibrated with Benzil (melting point $95{ }^{\circ} \mathrm{C}$ ). Once calibrated, a small amount of Fraction $\mathrm{E}$, was placed on the block at $75{ }^{\circ} \mathrm{C}$ and the crystals were slowly moved up to higher temperatures. The initial melting range was found to be 80 $85{ }^{\circ} \mathrm{C}$ with a slower movement of the sample resulting in a temperature of $82{ }^{\circ} \mathrm{C}$ which was when the crystals began to melt. A second method used an Electrothermal IA9200 series M383270/04 melting point apparatus. A capillary tube was loaded with a very small amount of Fraction $E$ which was tapped down to the bottom of the tube. The apparatus was set to 75 ${ }^{\circ} \mathrm{C}$ and the temperature increased at a rate of $1{ }^{\circ} \mathrm{C} \mathrm{min}^{-1}$. Fraction $\mathrm{E}$ was found to melt at 82 ${ }^{\circ} \mathrm{C}$. A second tube was loaded and the apparatus was set to $75{ }^{\circ} \mathrm{C}$ with a temperature increase of $0.2{ }^{\circ} \mathrm{C} \mathrm{min}^{-1}$. At $80^{\circ} \mathrm{C}$ the crystals started to melt, at $80.4{ }^{\circ} \mathrm{C}$ a meniscus formed in the capillary tube, at $81.2{ }^{\circ} \mathrm{C}$ all the solid had melted and at $82.0^{\circ} \mathrm{C}$ the liquid started to bubble.

\subsubsection{Gas chromatography-mass spectrometry (GC-MS)}

Aliquots of each Fractions A-I from column chromatography were derivatised with Sylon BTZ and analysed using gas chromatography-mass spectrometry (GC-MS; cf Rowland and Sutton, 2017). GC-MS was undertaken using an Agilent GC-MSD which had a 7890A gas chromatograph fitted with a $7683 \mathrm{~B}$ series autosampler coupled with a 5975A quadrupole 
This paper is a non-peer reviewed preprint submitted to EarthArXiv.

mass selective detector operated in electron impact mode with an ionisation voltage at $70 \mathrm{eV}$. $1 \mu \mathrm{L}$ of sample was injected (splitless mode; $250{ }^{\circ} \mathrm{C}$ ) onto a DB-5MS column (30 m x 0.25 $\mathrm{mm} \times 0.25 \mu \mathrm{m}$; Agilent Technologies UK Limited) with helium carrier gas (constant flow; 1.0 $\mathrm{mL} \mathrm{min}^{-1}$ ) and the oven programmed from $40-210^{\circ} \mathrm{C}$ at $10{ }^{\circ} \mathrm{C} \mathrm{min}^{-1}$ then from $210-300{ }^{\circ} \mathrm{C}$ at $5 \mathrm{C} \mathrm{min}^{-1}$ with a 10 minute hold. The transfer line temperature, ion source and quadrupole were operated at $280^{\circ} \mathrm{C}, 230^{\circ} \mathrm{C}$ and $150{ }^{\circ} \mathrm{C}$, respectively.

\subsubsection{Fourier-Transform Infrared (FT-IR) spectroscopy}

FTIR spectroscopy was carried out using a Bruker Alpha Platinum ATR instrument. The resolution was $4 \mathrm{~cm}^{-1}$. Data were collected from 4000 to $375 \mathrm{~cm}^{-1}$. Before any samples were analysed, the sample plate was cleaned with ethanol ( $3 \times 5$ drops). A drop of the sample was placed on the sample plate and then analysed.

\subsubsection{Nuclear Magnetic Resonance (NMR) spectroscopy}

NMR spectroscopy used approximately $25 \mathrm{mg}$ of Fraction $\mathrm{E}$, which was diluted in approximately in $2 \mathrm{~mL}$ of deuterated chloroform $\left(\mathrm{CDCl}_{3}\right) \cdot{ }^{1} \mathrm{H},{ }^{13} \mathrm{C}$ and DEPT spectra were obtained. The instrument used was a JEOL JNM-LA400 FT spectrometer. The field strength was 9.399163 Tesla at $400 \mathrm{MHz}$, the software was Delta NMR Software V.4.3.5. For ${ }^{1} \mathrm{H}$ NMR, 64 scans were taken, for ${ }^{13} \mathrm{C}$ and DEPT spectra, 8192 scans were taken.

\subsection{Photo-exposure of ambrein}

The photo-exposure apparatus used was essentially as described by Ali et al. (1995) and West and Rowland (2012). Thus, a Heraeus Original Hanau Suntest CPS instrument was fitted with a xenonstrahler xenon lamp and a bath cooled by an ATC K4 chiller unit. The bath held up to four quartz photo tubes and was filled with glycerol within $5 \mathrm{~cm}$ from the lip of the bath. The ATC K4 was switched on and set to $4{ }^{\circ} \mathrm{C}$ for 20 minutes before the xenon lamp was turned on; after that, the system was left for 1 hour to equilibrate. The temperature of the glycerol was the measured using a thermometer and a temperature probe.

Quartz photo test tubes were washed with DCM and allowed to dry. Approximately $50 \mathrm{mg}$ of ambrein (Fraction E) was accurately weighed into each of five pre-weighed $7 \mathrm{~mL}$ vials. A small amount of acetone was used to dissolve the ambrein by sonication ( $5 \mathrm{~min}$; Sweep; 30 $\left.{ }^{\circ} \mathrm{C}\right)$. A Pasteur pipette was used to transfer the dissolved ambrein into each of the four quartz phototubes and $30 \mathrm{~mL}$ of seawater was added to each of the tubes. Four $2 \mathrm{~mL}$ vials were weighed using a microbalance and approximately $0.5 \mathrm{mg}$ of $5,10,15,20$-tetraphenyl$21 \mathrm{H}, 23 \mathrm{H}$-porphine copper (II) was weighed accurately and added to each phototube by suspending it in acetone and transferring by Pasteur pipette. Each phototube was sealed with a Quick fit quartz lid and the phototubes sealed with Parafilm covered in tin foil. One 
This paper is a non-peer reviewed preprint submitted to EarthArXiv.

phototube (dark control) was covered with tin foil. The four phototubes were placed inside the Heraeus suntest instrument in the chilled bath. A fifth phototube with no added porphine did not go into the instrument. The temperature of the glycerol bath was measured every hour. Following 3 hours of exposure to the xenon lamp, one phototube was removed. After a further 3 hours exposure, the three remaining phototubes (two replicate $6 \mathrm{~h}$ exposures and the tube in tin foil as a dark control) were removed.

Once the phototubes had cooled to room temperature, a liquid-liquid extraction of the seawater was carried out. The foil over the lid was removed, the Parafilm was removed and the contents were each transferred to separating funnels. Each phototube was rinsed with DCM $(3 \times 5 \mathrm{~mL})$, each time adding the DCM to the separating funnel. A further $20 \mathrm{~mL}$ DCM was added to each separating funnel, which were shaken gently, and the pressure vented. The separating funnel was left to stand until two layers had formed, the bottom, DCM, layer was collected in a pre-weighed round bottom flask. A further $2 \times 20 \mathrm{~mL}$ of DCM was added to the aqueous phase to extract any remaining DCM soluble compounds. This was repeated for each phototube. Solvent volume was reduced to approximately $3 \mathrm{~mL}$ with rotary evaporation and the extract transferred to a pre-weighed $7 \mathrm{~mL}$ vial with washings $(3 \times 0.5 \mathrm{~mL}$; DCM). Solvent was removed by nitrogen blowdown $\left(40^{\circ} \mathrm{C}\right)$. The resulting mass was diluted with DCM to $10 \mathrm{mg} \mathrm{mL}^{-1}$ and a FTIR spectrum obtained; then $10 \mu \mathrm{L}$ of each diluted solution was added to separate GC vials and derivatised with Sylon BTZ (nom. $50 \mu \mathrm{L}, 70{ }^{\circ} \mathrm{C}, 1 \mathrm{~h}$ ) followed by addition of $1 \mathrm{~mL}$ of DCM. The solution were then analysed by GC-MS with a solvent delay of $17 \mathrm{~min}$. Other aliquots were examined by GC-MS without derivatisation.

\section{Results}

\subsection{Characterisation of ambrein}

Several of the column chromatography fractions contained ambrein (determined by GC-MS as the trimethylsilyl (TMS) ether; cf Rowland and Sutton 2017) with Fraction E (white crystals; $412 \mathrm{mg} ; ~ 55 \%$ by weight) containing most (TMS ether; $98.5 \%$ GC-MS purity; Figure S1). The mass spectrum was comparable to that published by Rowland and Sutton (2017) for ambrein TMS ether.

The melting point was determined in this study as $82{ }^{\circ} \mathrm{C}$ using Kofler block and melting point apparatus. Hardwick and Laws (1951) obtained colourless crystals by evaporation of the solvent over days and recorded a melting point of $83{ }^{\circ} \mathrm{C}$ (Table S1). Ambrein was also recrystallised from pentane resulting in white irregular flakes by Governo et al. (1977) and Ohloff et al. (1977). The melting points reported in the literature are shown in Table S1. 
This paper is a non-peer reviewed preprint submitted to EarthArXiv.

The FTIR spectrum obtained for Fraction E (Figure S2), was similar to published spectra for purified ambrein (e.g. Governo et al. 1977), with comparable transmittances, particularly at $887,1385,1455,1714$ and $3402 \mathrm{~cm}^{-1}$. However, the spectrum of pure ambrein (cf Governo et al., 1977) appears only to have been published in tablature form previously (e.g. Mori and Tamura, 1990), as far as we are aware. The transmittance at $3402 \mathrm{~cm}^{-1}$ was attributed to hydroxyl group stretching. The strong transmittances at 2930 and $2866 \mathrm{~cm}^{-1}$ were attributed to $\mathrm{C}-\mathrm{H}$ stretching in methyl and methylene groups and the transmittances at 1460 and 1386 $\mathrm{cm}^{-1}$ to the corresponding $\mathrm{C}-\mathrm{H}$ bending (Rowland and Sutton, 2017). A weak transmittance at $3067 \mathrm{~cm}^{-1}$ was attributed to $\mathrm{C}-\mathrm{H}$ stretching of unsaturation in ambrein. The transmittance at $1645 \mathrm{~cm}^{-1}$ was attributed to $\mathrm{C}=\mathrm{C}$ stretch. In the fingerprint region, the transmittances at 938 and $887 \mathrm{~cm}^{-1}$ were assigned to $\mathrm{C}-\mathrm{H}$ out of plane bends in ambergris with high ambrein contents (Rowland and Sutton, 2017).

Nuclear magnetic resonance (NMR) spectroscopy was also used to characterise the purified ambrein (Fraction E). Spectra (Figures S3-S5) were compared with those of synthetic and biosynthetic ambrein (e.g. Mori and Tamura, 1990; Ueda et al. 2013). Software was also used to predict the likely ${ }^{1} \mathrm{H}$ and ${ }^{13} \mathrm{C}$ NMR spectra (Engineering, 2018) for comparison with the acquired data (Figures S4 and S6). The ${ }^{1} \mathrm{H}$ and ${ }^{13} \mathrm{C}$ NMR spectra (Figures S3,S5) were comparable to that presented by Rowland et al. (2018) for ambrein in crude ambergris and by Ueda et al. (2013) for biosynthetic ambrein. Similar NMR spectra have also been published by earlier workers (e.g. Moniz and Hammond, 1996; Mori and Tamura, 1990).

In the ${ }^{13} \mathrm{C}$ spectrum obtained for Fraction E (Figure S5), the expected 30 resonances for ambrein were observed. The resonances observed at 149, 136, 125, 109 and 74 ppm were assigned by Rowland et al. (2018) and Ueda et al. (2013). The published spectrum (Ueda et al. 2013) identified several impurities, which were not seen in the Fraction E material. The predicted spectrum (Figure S6) was used to assign the resonances in the experimentally obtained spectrum (cf Ueda et al. 2013).

Last, a DEPT spectrum for ambrein was obtained (Figure S7). Ambrein is a $\mathrm{C}_{30}$ compound which has $11 \mathrm{CH}_{3} / \mathrm{CH}$ groups which are resonances that are positive ('up' as illustrated herein). It has $13 \mathrm{CH}_{2}$ groups which are negative ('down') in a DEPT spectrum and 6 quaternary carbons, which are absent in a DEPT spectrum. The carbon atoms labelled 6" and 4' (Figures S5, S6) are quaternary and therefore do not appear in the DEPT spectrum. Carbon 3' (CH group) is positive in the DEPT spectrum at $125 \mathrm{ppm}$. The carbon bonded to 6" 
This paper is a non-peer reviewed preprint submitted to EarthArXiv.

in the alkene $\left(\mathrm{C}=\mathrm{CH}_{2}\right)$ is due to a $\mathrm{CH}_{2}$ group, observed at $109 \mathrm{ppm}$. Last, carbon 2 (Figure S7) is also quaternary and is absent in the DEPT spectrum. The three resonances at 54,56 and $62 \mathrm{ppm}$ are confirmed to be due to $\mathrm{CH}_{3}$ or $\mathrm{CH}$ groups by the DEPT spectrum. This is a strong indication they are due to carbons 9, 5 and 17 (Figure S7), as supported by both the predicted spectrum and the DEPT spectrum. Resonances between 10 - 50 ppm (Figure S7) show that there is a further 13 negative $\mathrm{CH}_{2}$ groups and 8 positive $\mathrm{CH}_{3} / \mathrm{CH}$ groups. Since this totals more groups than expected, the DEPT spectrum also indicates the presence of minor impurities, consistent with the $98.5 \%$ purity determined by GC-MS.

In summary, all of the chromatographic, spectroscopic and melting point data support assignment of Fraction $\mathrm{E}$ as $>98 \%$ pure ambrein. This was deemed a suitable substrate for photo-exposure experiments.

\subsection{Photo-exposure of ambrein}

GC-MS analysis was carried out of triplicate samples of extracts of ambrein in seawater for 0 $\mathrm{h}$ exposure, a single sample for $3 \mathrm{~h}$ exposure, duplicates for the $6 \mathrm{~h}$ exposure and a sample representing a dark control $(6 \mathrm{~h})$. Extracts were each analysed twice by GC-MS: once after derivatisation with Sylon reagent (Rowland and Sutton, 2017) with a 17 minute solvent delay to avoid detection of the Sylon derivatisation by-products but to allow determination of unreacted ambrein as the TMS ether (Figure 1) and to avoid formation of ambratriene artefacts (Governo et al., 1977); second, with a solvent delay of $8 \mathrm{~min}$ and no derivatisation (e.g. Figures 2, 3), in order to enhance detection of more volatile and semi-volatile products. Formation of ambratrienes under the latter conditions, was unavoidable, thus analysis focussed on the more volatile products (cf Figure 2 et seq.).

Determined by GC-MS of the derivatised extracts, the proportion of unoxidised ambrein decreased by about $40 \%$ of the initial amount within about $3 \mathrm{~h}$ exposure (Figure 1). Further exposure (a further $3 \mathrm{~h} ; 6 \mathrm{~h}$ total) produced relatively little further change (Figure 1). Interestingly, a similar decrease was observed in the dark control (orange point, Figure 1). The experiment aimed to photo-oxidise ambrein under realistic simulated environmental conditions. The Heraeus suntest instrument exposed the sample to about $500 \mathrm{~W} \mathrm{~m}^{-2}$ (West and Rowland, 2012). It has been estimated that about $3 \mathrm{~h}$ under this xenon lamp, which has a spectrum close to that of natural sunlight (West and Rowland, 2012), equates to about 24 h Florida midday midsummer sunlight (Ali, 1994). Therefore, herein, the sample exposed to $3 \mathrm{~h}$ is equivalent to a natural exposure of about $24 \mathrm{~h}$ of Florida daytime sunlight and the samples exposed for $6 \mathrm{~h}$ exposure to in excess of $48 \mathrm{~h}$ peak Florida daytime sunlight. Also, 
This paper is a non-peer reviewed preprint submitted to EarthArXiv.

the ambrein was exposed in the presence of a copper porphyrin $(5,10,15,20$-tetraphenyl$21 \mathrm{H}, 23 \mathrm{H}$-porphine copper (II)), forms of which has been found in ambergris, probably as a relict of the haemolymph of the squid diet of the sperm whale (Lederer, 1949). This has been suggested previously to act as a natural photosensitiser (Lederer, 1949). These conditions may have led to an efficient oxidation of the ambrein; however, the similar result observed for the (albeit single) dark control sample, suggests the large quantitative changes may have been to an autooxidative, perhaps thermal, processes.

Previous experiments in which ambrein was exposed to light produced about $0.3 \%$ detectable compounds, many of which were volatile (Mookerjee and Patel, 1977). Ruzicka et al., (1948) also suggested that about $0.3 \%$ degradation products were normally found in ambergris. We therefore conducted an assay of the amounts of products extractable from the seawater matrix and detectable by GC-MS, after exposure. Likely our methods, which did not examine the headspace of the phototubes after exposure, did not recover most of the volatile products quantitatively.

The peak area from the GC-MS chromatogram for a component identified from comparison of the mass spectrum with that of a NIST library, as ambroxan (note ambroxan has many other synonyms), was used to determine the total concentration of the GC-MS detectable products of the extracts. In the $3 \mathrm{~h}$ and $6 \mathrm{~h}$ exposed samples, the ambroxan concentration was $\sim 0.002 \mathrm{mg} \mathrm{mL}^{-1}$. This represents about $0.1 \%$ by weight of the ambrein present initially. Summation of the GC-MS peak heights of all of the components detectable under these conditions, suggested a total of about $1 \%$ products. This clearly does not account for the $40 \%$ losses observed, even in the dark control (Figure 1). Undetectable volatile or non-GCMS detectable products may have formed and these may account for the major losses.

The chromatograms of each of the extracts from the photo-oxidation experiments (Figure 2) shown on a scale relative to each other nonetheless indicate that there are differences in the detectable products between the exposed samples $\left(t=3,6_{1}, 6_{2} h\right)$ and that of the dark control (Figures 2,3). There is also a smaller difference between the two $6 \mathrm{~h}$ replicates (Figure 3; yellow and light blue).

A compound eluting at 11.06 min retention time (retention index 1398) produced a mass spectrum (Figure 4) with an $85 \%$ match to that of the NIST mass spectrum of $\mathrm{y}$ dihydroionone. However, Mookherjee and Patel (1977) showed that $\mathrm{Y}$-dihydroionone may undergo cyclisation into a-ambrinol under some experimental conditions. It is therefore possible that $\alpha$-ambrinol was a photo-oxidation product, but may have thermally degraded to 
This paper is a non-peer reviewed preprint submitted to EarthArXiv.

$\mathrm{Y}$-dihydroionone in the hot GC-MS injector during analysis. However, it is more than likely that the $\mathrm{y}$-dihydroionone was indeed a photo-oxidation product, as reported previously (Shen et al., 2007). Alternatively, both $\alpha$-ambrinol and $\gamma$-dihydroionone may have been produced by photooxidation, with only the latter detectable under the conditions used.

At 15.50 min retention time (retention index 1775; cf published retention index of 1765 for a different stationary phase; Rowland and Sutton, 2017), a compound with a mass spectrum (Figure 5) comparable to the NIST mass spectrum of ambroxan was present. The spectrum and retention position also compared well with that of a sample of authentic ambroxan, examined herein. Thus, the experiment has shown that under the experimental conditions, ambrein does photodegrade to ambroxan. No additional ambroxan was detected in the dark control compared to the $0 \mathrm{~h}$ sample (Figures 2,3); the proportion of ambroxan in the dark control (orange, Figure 3) was within the uncertainty of the value obtained by triplicate measurements of the $\mathrm{t}=0 \mathrm{~h}$ sample; therefore ambrein was not undergoing auto-oxidation to ambroxan under the conditions used.

At 16.32 min retention time (retention index 1853) a compound with a mass spectrum (Figure 6) which matched (98\%) that of a NIST database spectrum of a tricyclic enol ether, was present. No molecular ion at $m / z 266$ was observed, but an ion $m / z 248\left(\mathrm{M}^{+}-\mathrm{H}_{2} \mathrm{O}\right)$, was present. The same compound was also determined by Ohloff et al., (1977) to be a tricyclic enol ether, which was found as an odourless component in ambergris.

The mass spectrum of a component which eluted at 19.43 min (retention index 2138) matched somewhat (85\%) that of 13-epoxy-14, 15, 16-trinorlabdan-13-one. The spectrum was found in the NIST database and also reported by Awano et al., (2005). Ambrein perhaps photo oxidised to the tricyclic enol ether, then further oxidation might have formed the lactone. However, the spectral match was not absolute, so some doubt about this assignment must remain.

Many other minor components of the exposed ambrein, were detected by GC-MS (Figure 2), but these were not identified, nor explored further herein. Awano et al., (2005) identified 31 constituents in ambergris tincture but several were artefactual. Shen et al., (2007) and Ohloff et al., (1977) suggested that many of the non-artefactual products derived from photooxidation of ambrein via a number of possible oxygenated $\mathrm{C}_{30}$ intermediates (Figures S8, S9). These were not detected in the present study. 
This paper is a non-peer reviewed preprint submitted to EarthArXiv.

\section{Conclusions}

Experiments were conducted herein, in which ambrein (>98\% pure by GC-MS, FTIR and NMR spectroscopy) was isolated from jetsam ambergris. Ambrein in seawater (1666 mg L-1), containing a photosensitiser a form of which is found naturally in ambergris $(5,10,15,20$ tetraphenyl-21H, $23 \mathrm{H}$-porphine copper (II); $1 \%$ of ambrein $\mathrm{w} / \mathrm{w}$ ), was exposed to simulated natural sunlight for experimental periods of up to $6 \mathrm{~h}$ (approximately equivalent to $48 \mathrm{~h}$ natural exposure, Florida midday, mid-summer sunlight). Dark controls were treated in the same manner, without exposure to light.

After $6 \mathrm{~h}$ exposure to light, total reaction products detectable by gas chromatography-mass spectrometry of solvent extracts, comprised about $1 \%$ by mass of the initial ambrein. However, unaccounted-for mass losses of ambrein were much greater than this ( $50 \%)$, even in the dark, suggesting major losses of volatile products and/or non-recovery of some oxidation products by the extraction methods used.

Identified photo-oxidation products included ambroxan, $\mathrm{y}$-dihydroionone, a tricyclic enol ether and possibly a related lactone. All of these have been reported previously in photoexposures of ambrein made under, arguably, less environmentally realistic conditions. The products were not observed in dark controls however, suggesting they were indeed products of photo-oxidation.

\section{Acknowledgements}

We are grateful to J. Smith and A. Wells, of Ambergris Connect Ltd, for donation of the sample of jetsam ambergris taken from a boulder collected in Chiloé Island, Chile, by $\mathrm{T}$. Helle Pessot, S. Mansilla Bastías and N. Wolff Reinarz (Universidad Austral de Chile).

\section{References}

Ali, L.N. (1994) The Dissolution and Photodegradation of Kuwait Crude Oil in Seawater. Ph.D thesis, University of Plymouth. 
This paper is a non-peer reviewed preprint submitted to EarthArXiv.

Ali, L.N., Mantoura, R.F. \& Rowland, S.J. (1995). The dissolution and photodegradation of Kuwaiti crude oil in seawater. Part 2: a laboratory photodegradation apparatus and photodegradation kinetics of a model seawater soluble hydrocarbon (phenanthrene). Marine Environmental Research 40, 319-335.

Anon. (1976). Ambergris tincture. Food and Cosmetics Technology 14 (Supplement) 675676.

Awano, K., Ishizaki, S., Takazani, O. \& Kitahara, T. (2005). Analysis of ambergris tincture. Flavour \& Fragrance Journal, 20, 18-21.

Clarke, R. (2006). The origin of ambergris. LAJAM, 5, 7-21.

Engineering (2018) Nmrdb.org. [Online] Available at http://www.nmrdb.org/new predictor/index.shtml?v=v2.79.1 [Accessed 19 April 2018].

Governo, T. F., Alessandro, R. T. \& Prager, M. J. (1977). Gas-Liquid Chromatographic-Mass Spectrometric Detection and Identification of Ambergris. JAOAC, 60, 160-164.

Hardwick, P. J. \& Laws, E. Q. (1951). The Identification of Ambergris. Analyst, 76, 662-664.

Lederer, E. (1949). Chemistry and Biochemistry of Some Mammalian Secretions and Excretions. Journal of the Chemical Society, 2115 - 2125.

Moniz, G.A. \& Hammond, G.B. 1996. Identification of ambergris from the New Bedford whaling museum by nuclear magnetic resonance spectroscopy. JAOAC Int. 79, 423-425.

Mookherjee, B. \& Patel, R. (1977). Isolation and identification of volatile constituents of tincture ambergris. 7th International Congress of Essential Oils, 136, 479-482.

Mori, K. \& Tamura, H. (1990). Triterpenoid total synthesis I. Synthesis of ambrein and Ambrox®. Liebigs Ann. Chem. 361-368.

Ohloff, G., Schulte-Elte, K. \& Muller, B. (1977). Formation of ambergris odorants from ambrein under simulated natural conditions. Helv. Chim. Acta, 60, 2763-2766. 
This paper is a non-peer reviewed preprint submitted to EarthArXiv.

Rowland, S.J. \& Sutton, P.A. (2017). Chromatographic and spectral studies of jetsam and archived ambergris. Natural Product Research 31, 1752-1757.

Rowland, S.J., Sutton, P.A., Belt, S.T., Fitzsimmons-Thoss, V. \& Scarlett, A.G. (2018).

Further spectral and chromatographic studies of ambergris. Natural Product Research. [doi: 10.1080/14786419.2018.1428599].

Ruzicka, L., Seidel, C.F. \& Pfeiffer, M. (1948). Über die flüchtigen Bestandteile des grauen Ambra. 1. Mitteilung. Isolierung von Dihydro-y-jonon. Helvetica Chimica Acta 31, 827.

Shen, Y.C., Cheng, S.Y., Kuo, Y.H., Hwang, T.L., Chiang, M.Y. \& Khalil, A.T. (2007). Chemical Transformation and Biological Activities of Ambrein, a Major Product of Ambergris from Physeter macrocephalus (Sperm Whale). J. Nat. Prod., 70, 147-153

Sutton P.A. and Rowland S.J. (2012). High temperature gas chromatography-time-of-flightmass spectrometry (HTGC-ToF-MS) for high-boiling compounds. Journal of Chromatography A 1243, 69-80.

Ueda, D., Hoshino, T. \& Sato, T. (2013). Cyclization of Squalene from Both Termini: Identification of an Onoceroid Synthase and Enzymatic Synthesis of Ambrein. J. Am. Chem. Soc., $135,18335-18338$.

West, C. E. \& Rowland S. J. (2012) Aqueous phototransformation of diazepam and related human metabolites under simulated sunlight. Environmental Science \& Technology 46, 4749-4756. 
This paper is a non-peer reviewed preprint submitted to EarthArXiv.

Figure 1. Percent of ambrein (I) during 6h exposure with (blue dots) and without (orange dot, dark control) light, relative to initial concentrations.. Sample points indicate results of triplicate $(0 \mathrm{~h})$ single ( $3 \mathrm{~h}, 6 \mathrm{~h}$ dark control) or duplicate ( $6 \mathrm{~h}$ exposed) samples. Values were determined by GC-MS in DCM extracts after derivatisation.

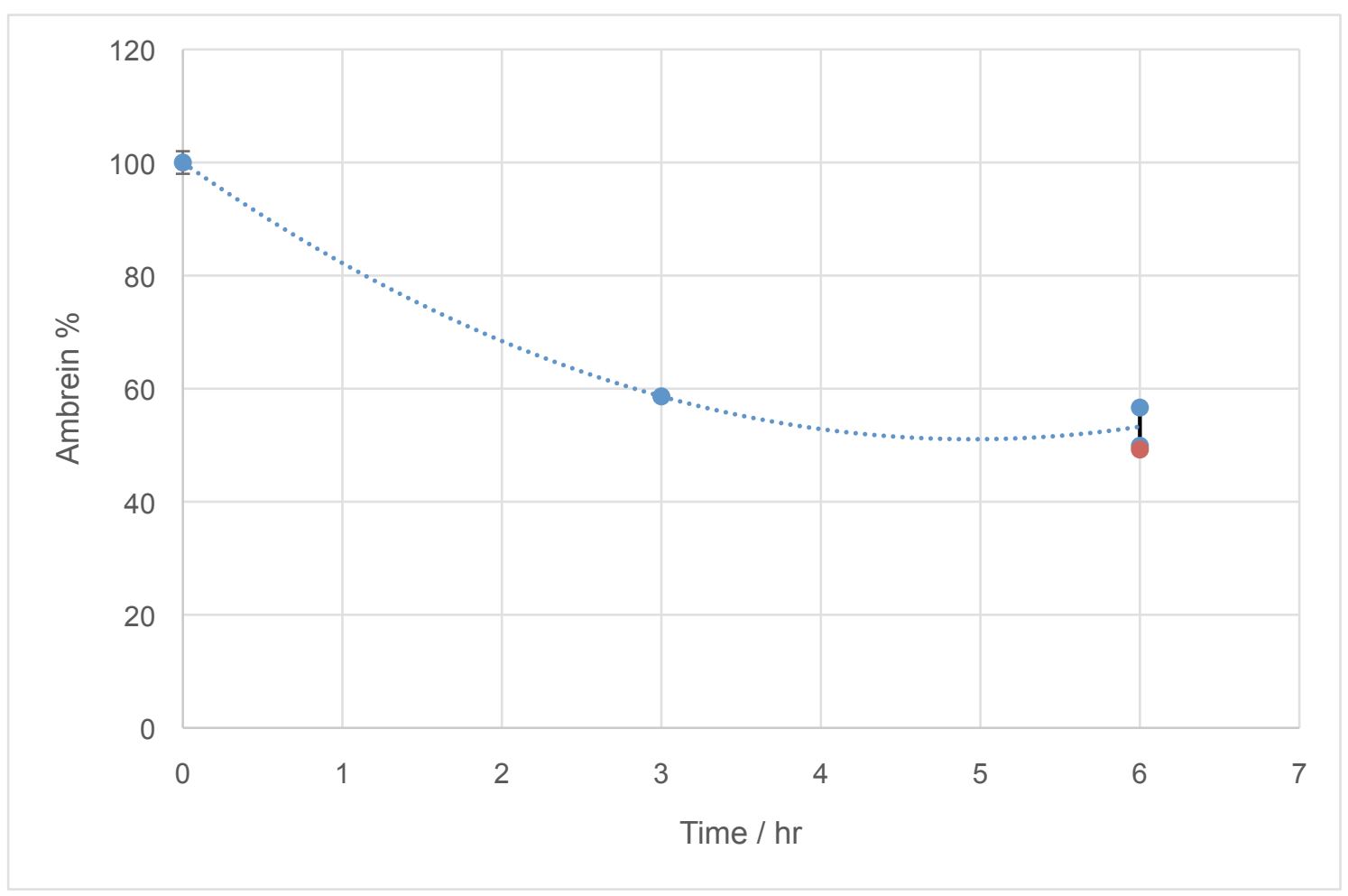

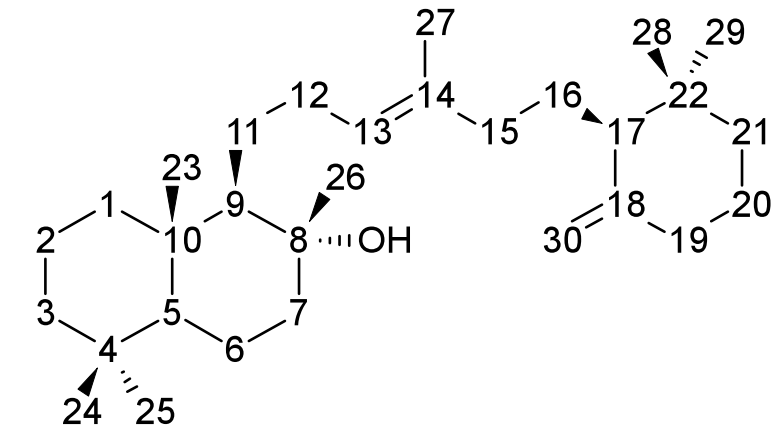

(I) 
This paper is a non-peer reviewed preprint submitted to EarthArXiv.

Figure 2. GC-MS total ion current chromatograms showing detectable semi-volatile (nonderivatised) products of exposure of ambrein, in seawater in presence of a natural photosensitiser, during $6 \mathrm{~h}$ exposure to $500 \mathrm{~W} \mathrm{~m}^{-2}$ simulated natural sunlight Peaks at retention times $11.06,15.50,16.32$ and 19.43 minutes were identified or tentatively identified, as $\mathrm{Y}$-dihydroionone, ambroxan, a tricyclic enol ether and a tricyclic lactone, 13-epoxy-14, 15, 16-trinorlabdan-13-one, respectively. All chromatograms are shown on same relative scale.

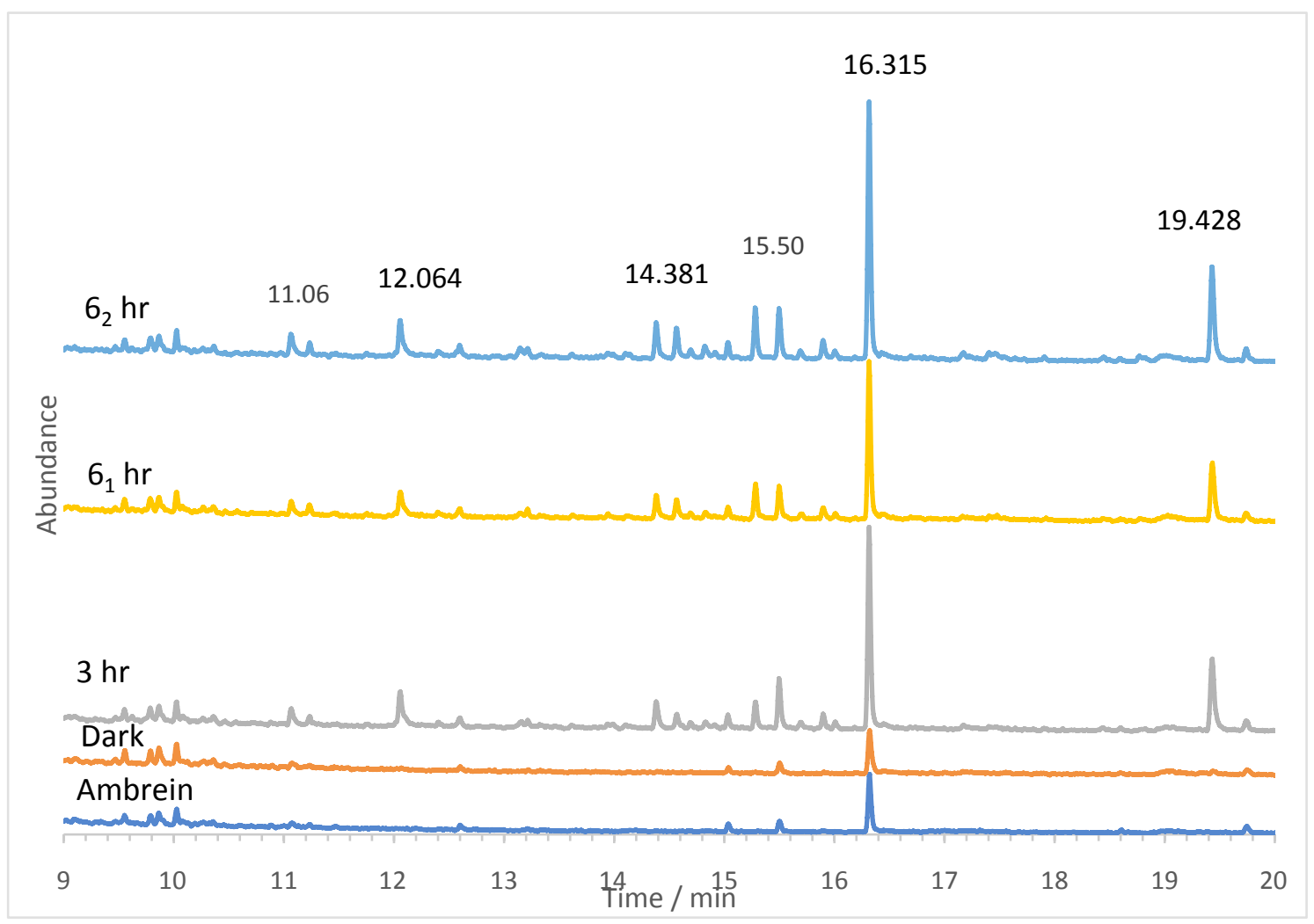


This paper is a non-peer reviewed preprint submitted to EarthArXiv.

Figure 3. Relative production (GC-MS peak areas, arbitrary units) of ambroxan by photoexposure of ambrein. Sample points indicate results of triplicate $(0 \mathrm{~h})$ single $(3 \mathrm{~h}, 6 \mathrm{~h}$ dark control (orange)) or duplicate ( $6 \mathrm{~h}$ exposed) samples. Values were determined by GC-MS in DCM extracts without derivatisation. A polynomial with order 3 has been used.

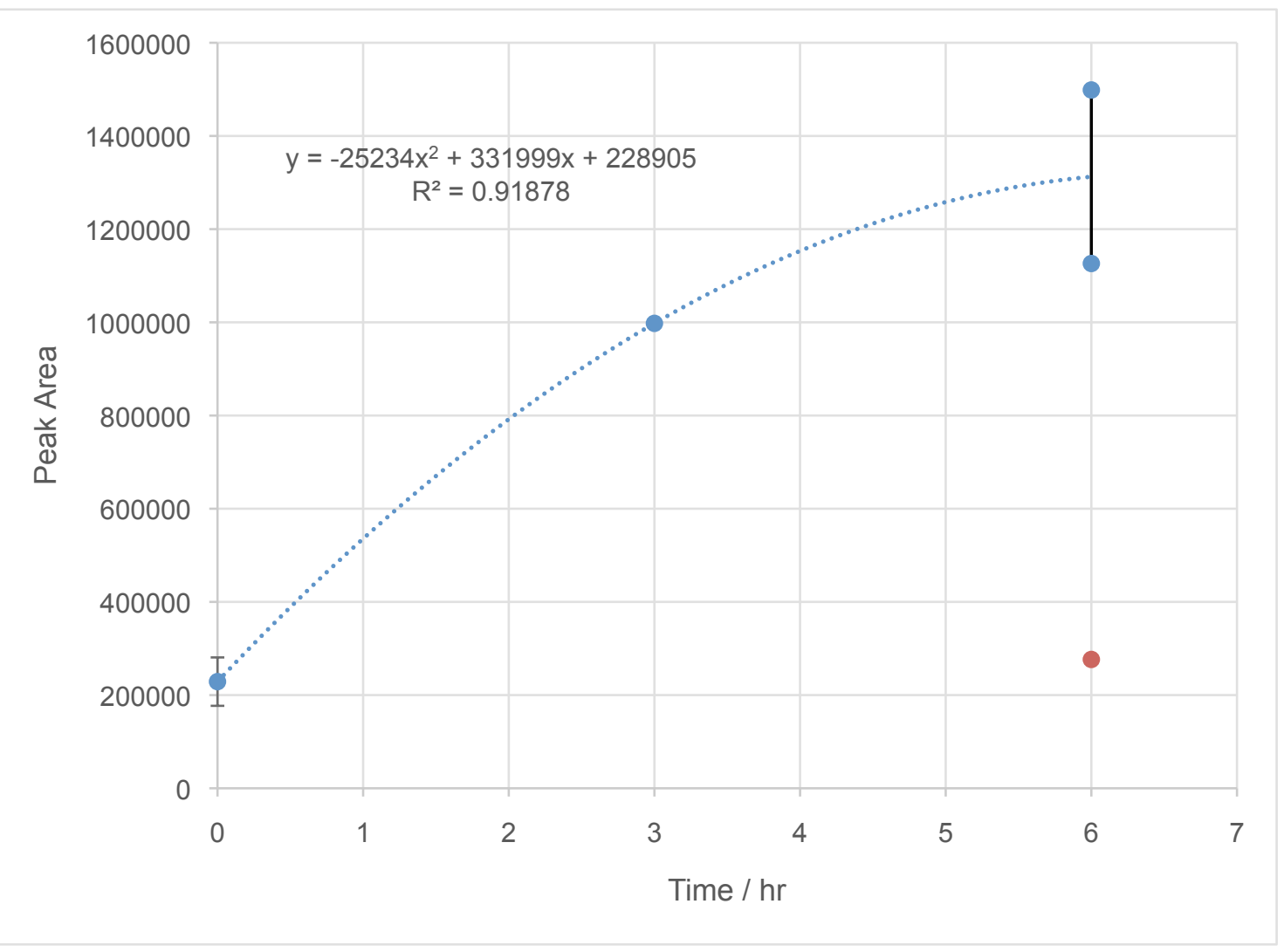


This paper is a non-peer reviewed preprint submitted to EarthArXiv.

Figure 4. Structures and interconversion of $\gamma$-dihydroionone and $\alpha$-ambrinol (Mookerjee and Patel, 1977) and mass spectrum of component tentatively assigned as $\gamma$-dihydroionone (retention time 11.06 minutes, Figure 2).<smiles>C=C1CCCC(C)(C)[C@H]1CCC(C)=O</smiles>

$\gamma$-dihydroionone

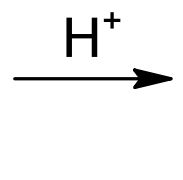

$\mathrm{HO}$

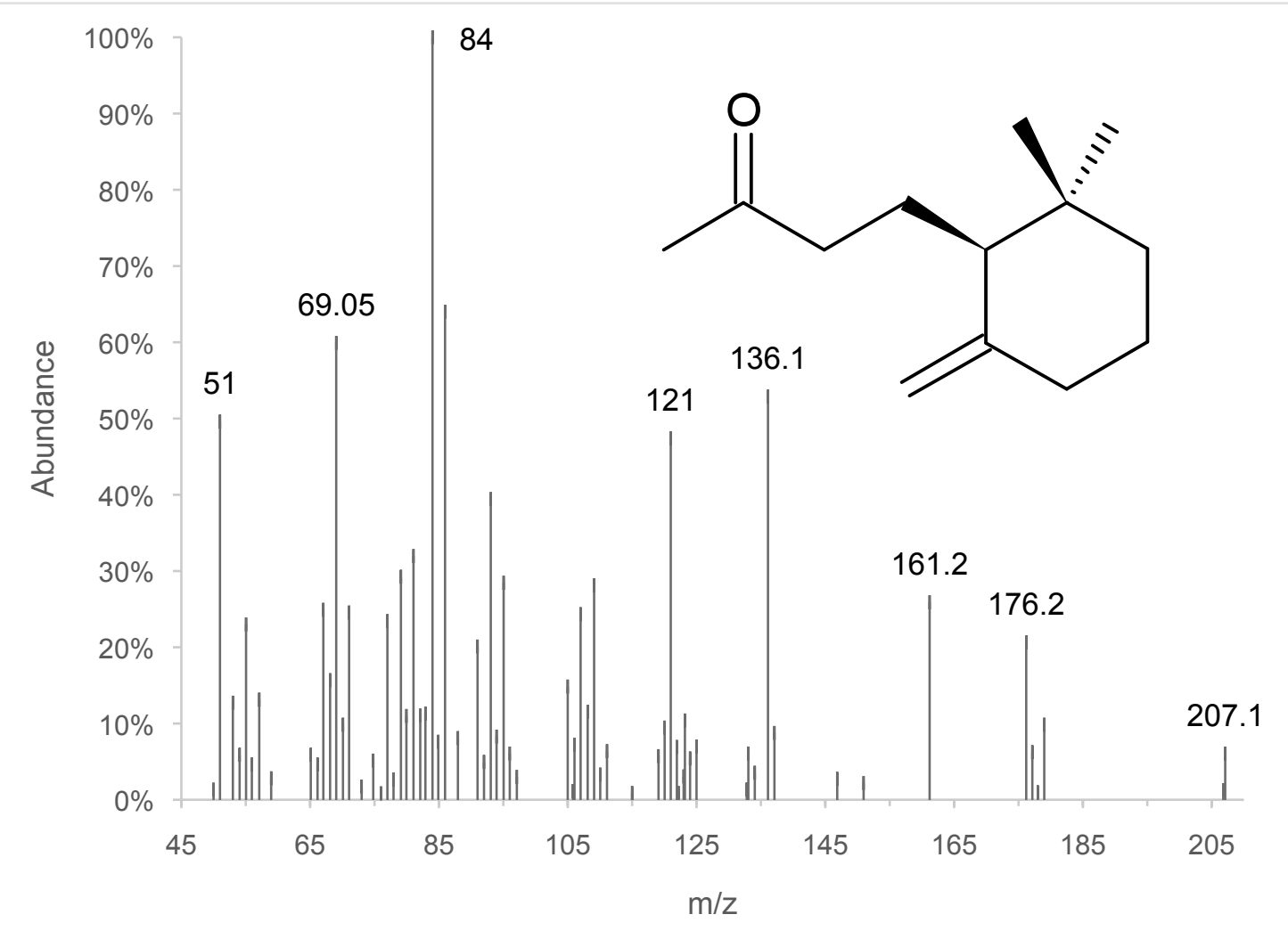


This paper is a non-peer reviewed preprint submitted to EarthArXiv.

Figure 5. Mass spectrum and structure of component assigned as ambroxan (retention time 15.50 minutes, Figure 2)

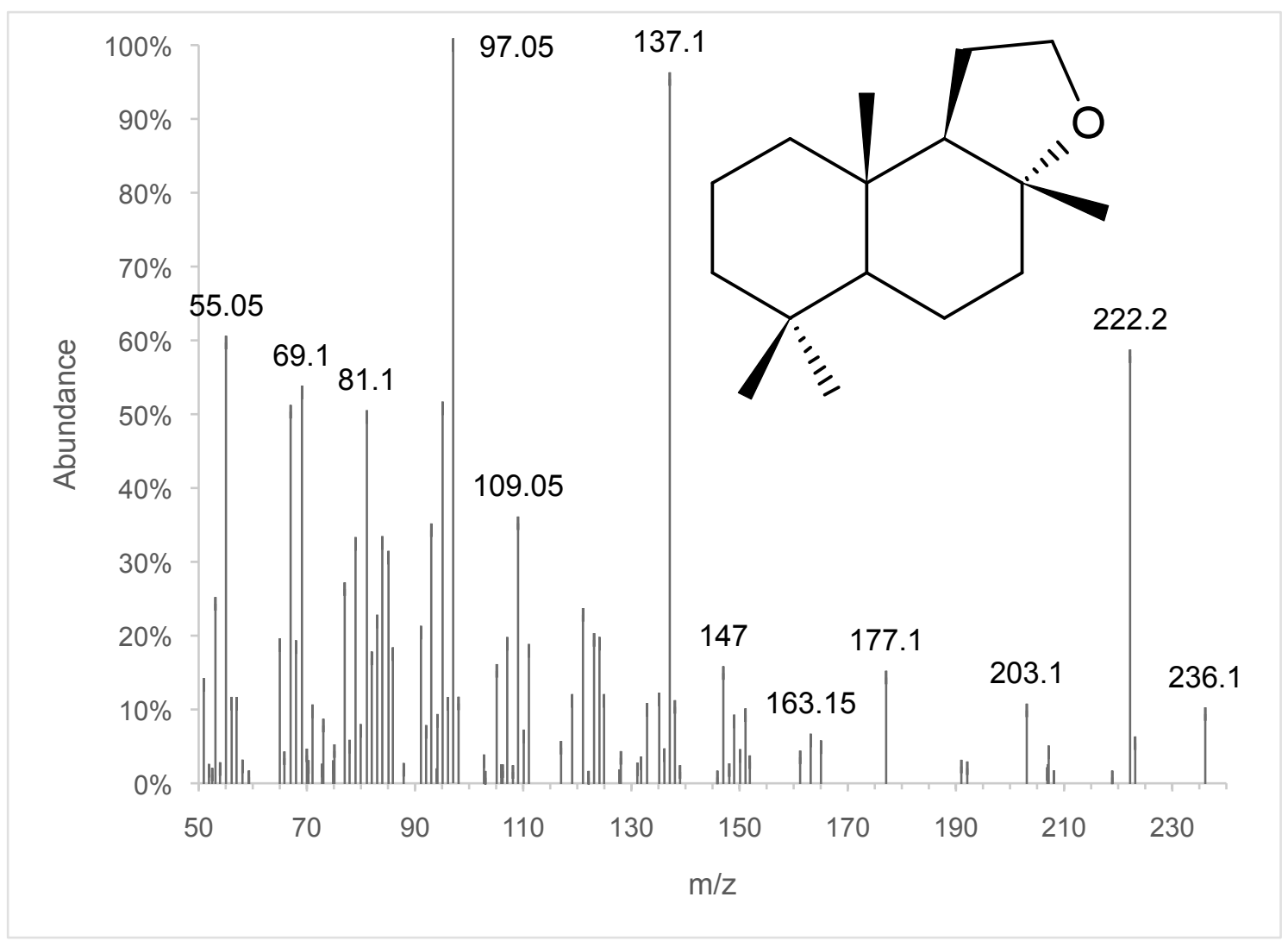


This paper is a non-peer reviewed preprint submitted to EarthArXiv.

Figure 6. Mass spectrum and structure of component assigned as a tricyclic enol ether (retention time 16.32 minutes, Figure 2; cf Ohloff et al., 1977)

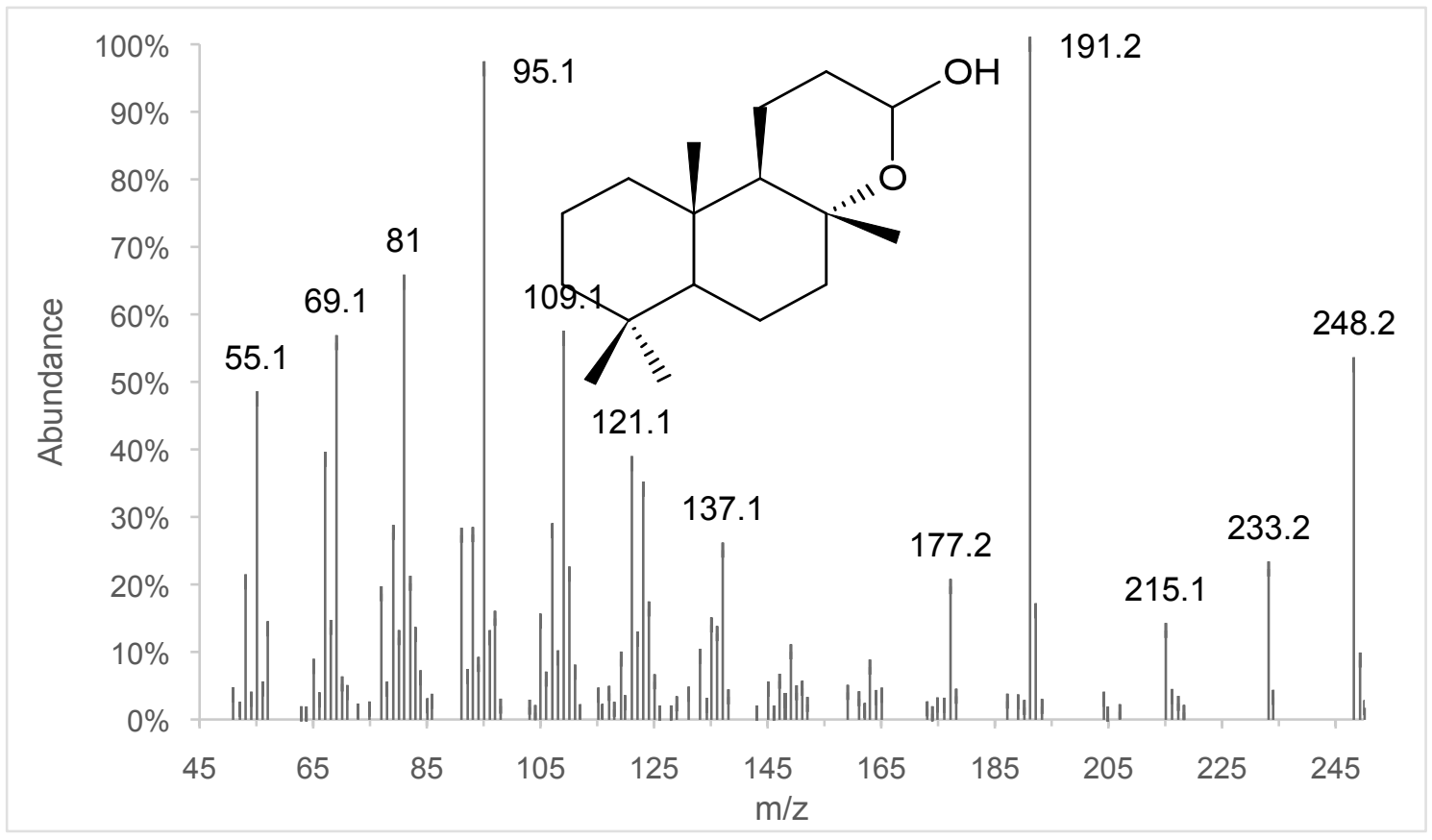

Table 1. Sequential solvents used for the silica column chromatographic fractionation of ambergris. * Sum of fractions I-K.

\begin{tabular}{|c|l|c|c|}
\hline Fraction & \multicolumn{1}{|c|}{ Solvent } & Mass (g) & Volume $(\mathbf{m L})$ \\
\hline A & $n$-hexane & 0.002 & 40 \\
\hline B & $n$-hexane & 0.001 & 40 \\
\hline C & $n$-hexane:diethyl ether $(9: 1, \mathrm{v} / \mathrm{v})$ & 0.002 & 40 \\
\hline D & $n$-hexane:diethyl ether $(4: 1, \mathrm{v} / \mathrm{v})$ & 0.047 & 40 \\
\hline E & $n$-hexane:diethyl ether $(1: 1, \mathrm{v} / \mathrm{v})$ & 0.412 & 40 \\
\hline F & $n$-hexane:diethyl ether $(1: 1, \mathrm{v} / \mathrm{v})$ & 0.102 & 40 \\
\hline G & Diethyl ether & 0.040 & 40 \\
\hline H & Diethyl ether & 0.029 & 40 \\
\hline I & Toluene & $0.008^{*}$ & 40 \\
\hline J & Methanol & $0.008^{*}$ & 40 \\
\hline K & Methanol & $0.008^{*}$ & 40 \\
\hline
\end{tabular}


This paper is a non-peer reviewed preprint submitted to EarthArXiv.

\section{Supplementary Material}

Table S1 Melting point data for isolated and synthetic ambrein.

Reference

Hardwick \& Laws, 1951

Governo et al. 1977

Mori \& Tamura, 1990

Ohloff et al. 1977

Lederer, 1949

This Study

\section{Melting Point $\left({ }^{\circ} \mathrm{C}\right)$}

83

$79.5-80.5$

$80.5-82$

$81-82$

83

82

\section{Notes}

Colourless crystals

Uncorrected values

Synthesised compound 
This paper is a non-peer reviewed preprint submitted to EarthArXiv.

Figure S1. GC-MS total ion current chromatogram of derivatised Fraction E from the column chromatography of ambergris extract. The peak due to ambrein TMS ether at 31.872 minutes produced $98.5 \%$ of the recorded response.

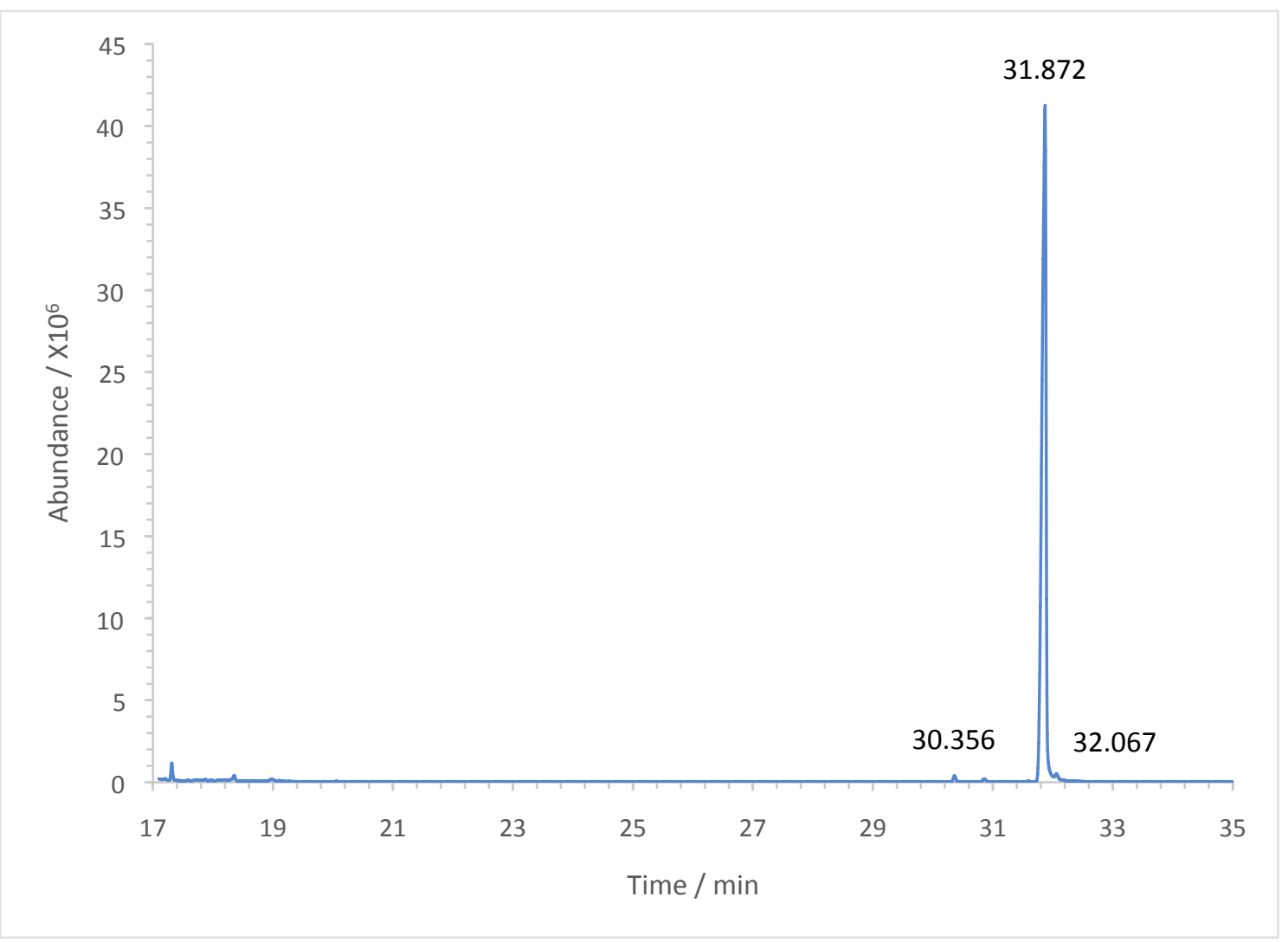


This paper is a non-peer reviewed preprint submitted to EarthArXiv.

Figure S2 FTIR spectrum of Fraction E from the column chromatography of ambergris extract.

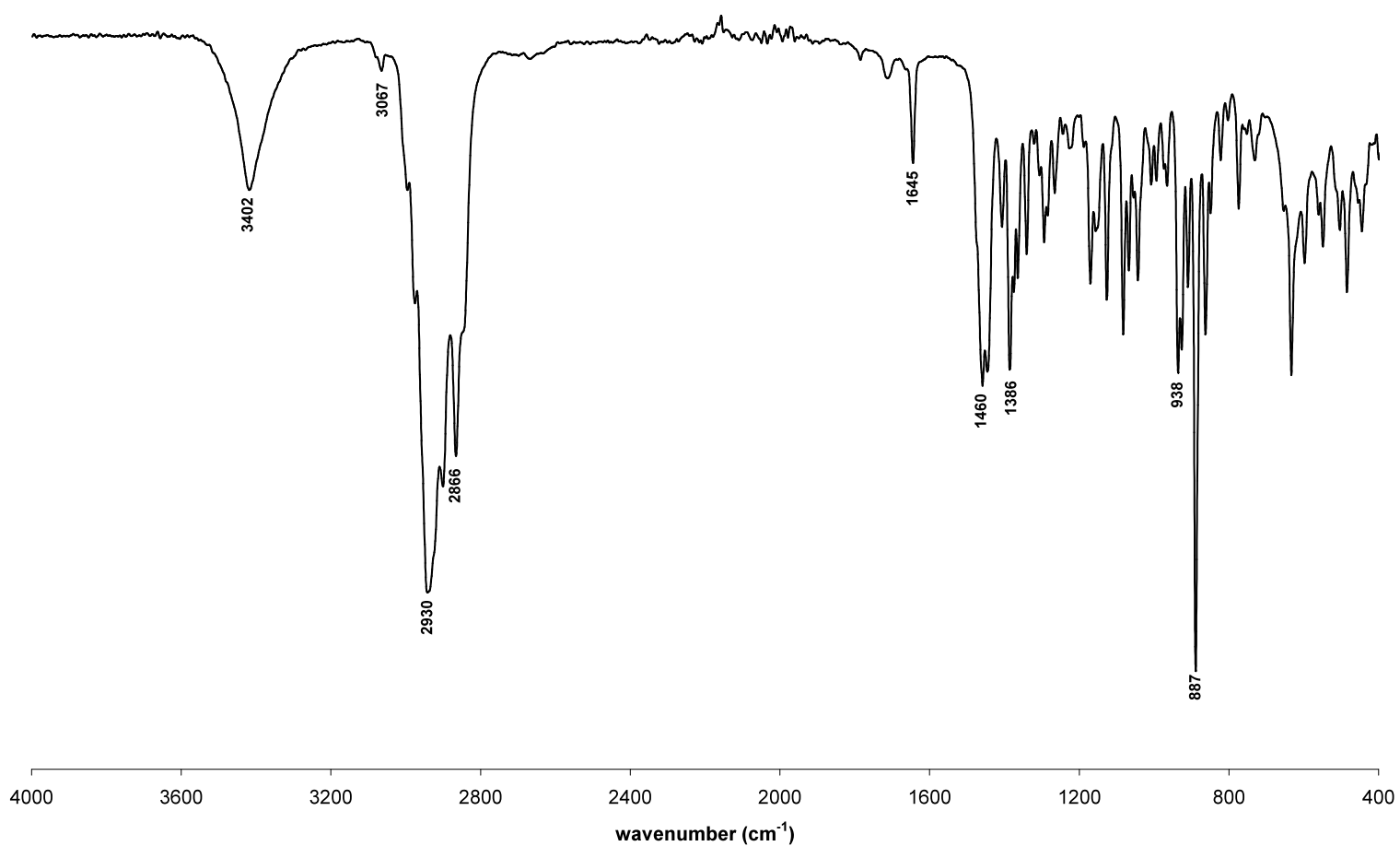


This paper is a non-peer reviewed preprint submitted to EarthArXiv.

Figure S3 ${ }^{1} \mathrm{H}$ NMR spectrum of Fraction E from the column chromatography of ambergris extract.
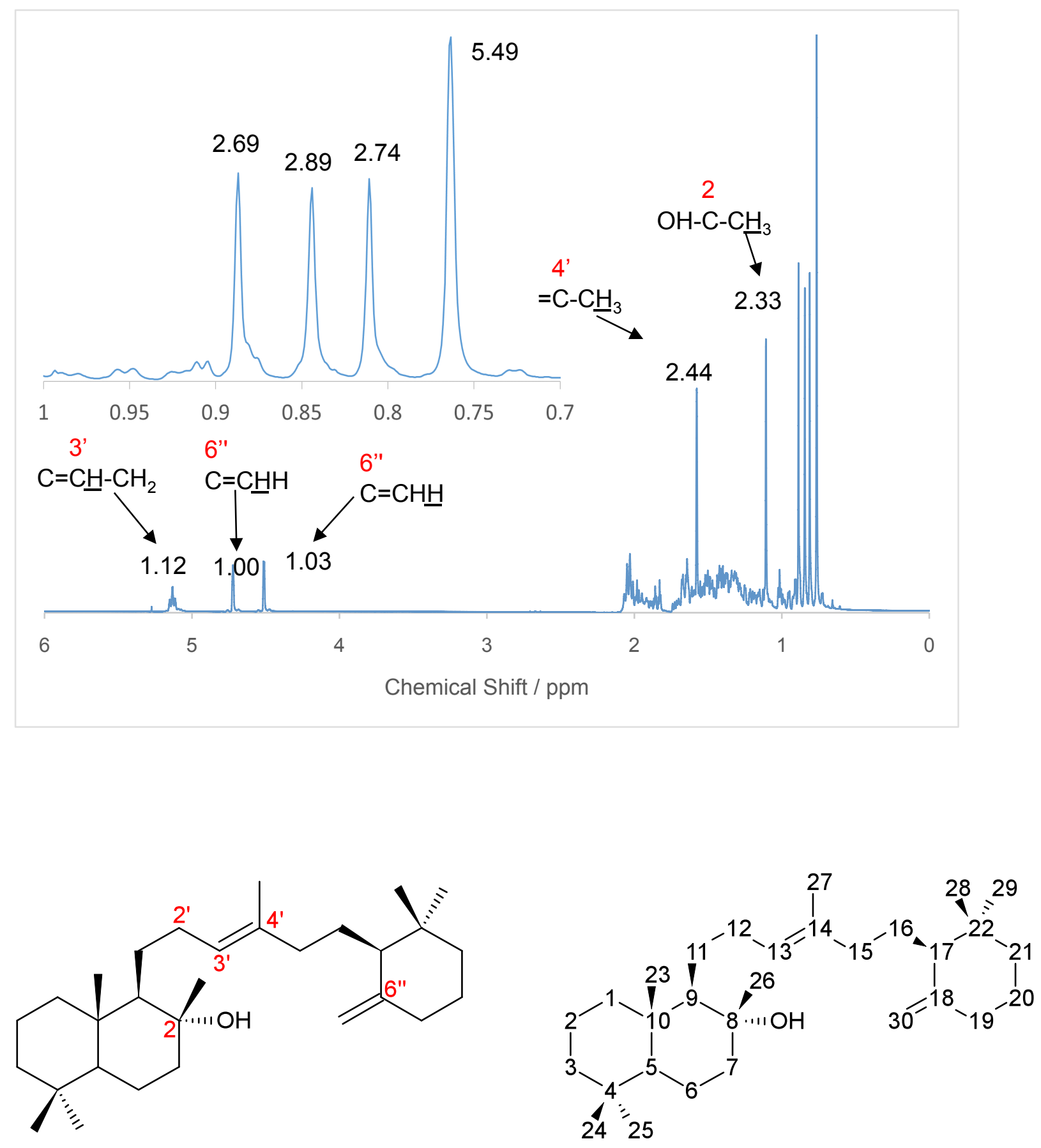
This paper is a non-peer reviewed preprint submitted to EarthArXiv.

Figure S4 Predicted ${ }^{1} \mathrm{H}$ NMR spectrum of ambrein (Engineering 2018).
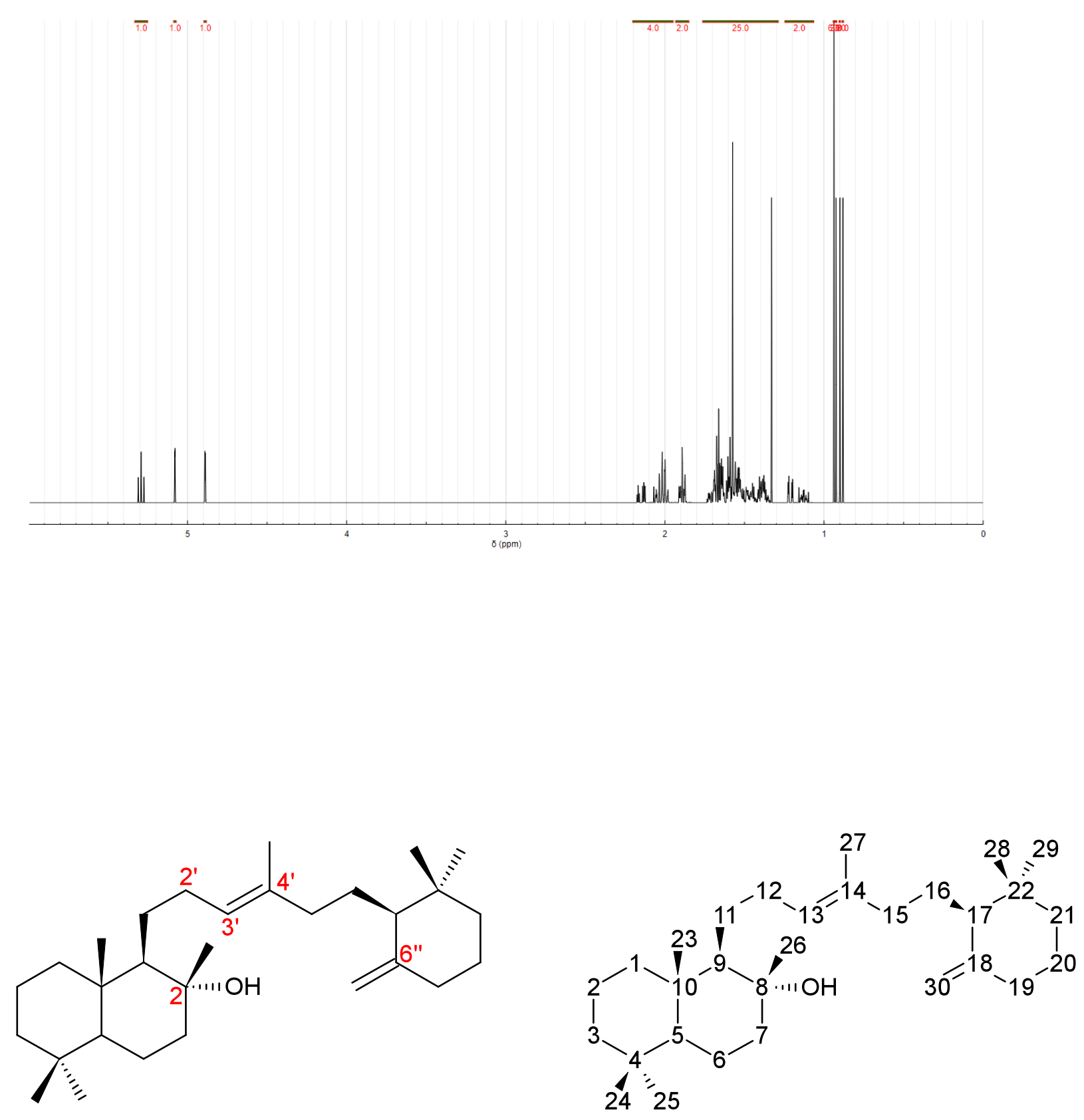
This paper is a non-peer reviewed preprint submitted to EarthArXiv.

Figure S5 ${ }^{13} \mathrm{C}$ NMR spectrum of Fraction $\mathrm{E}$ from the column chromatography of ambergris extract. The arrowed, bracketed, group comprises peaks due to 22 further resonances (i.e. 30 in all).

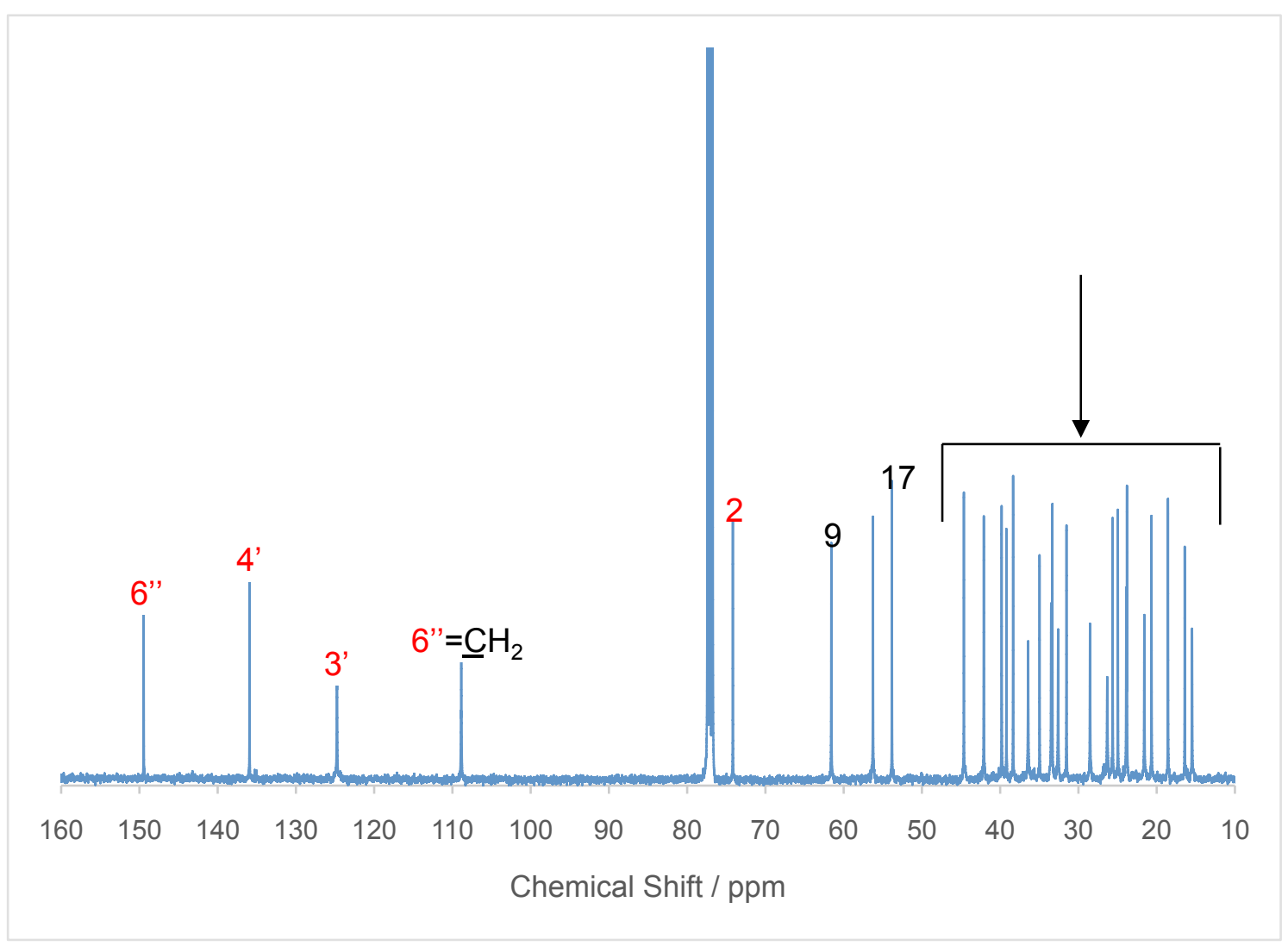

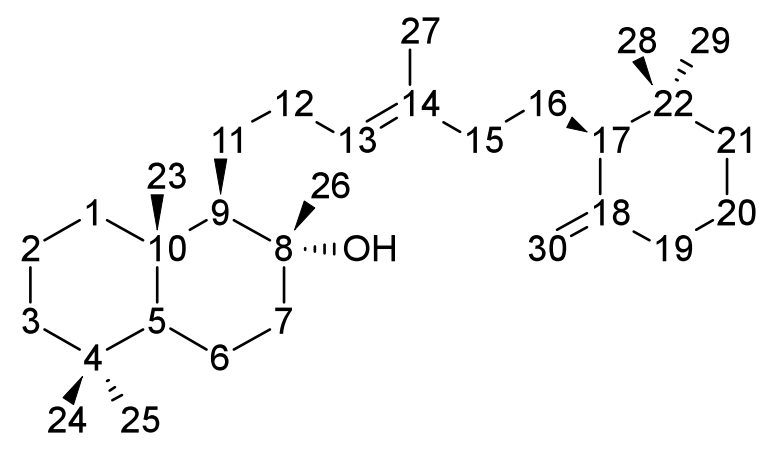


This paper is a non-peer reviewed preprint submitted to EarthArXiv.

Figure S6 Predicted ${ }^{13} \mathrm{C}$ NMR spectrum of ambrein (Engineering 2018).
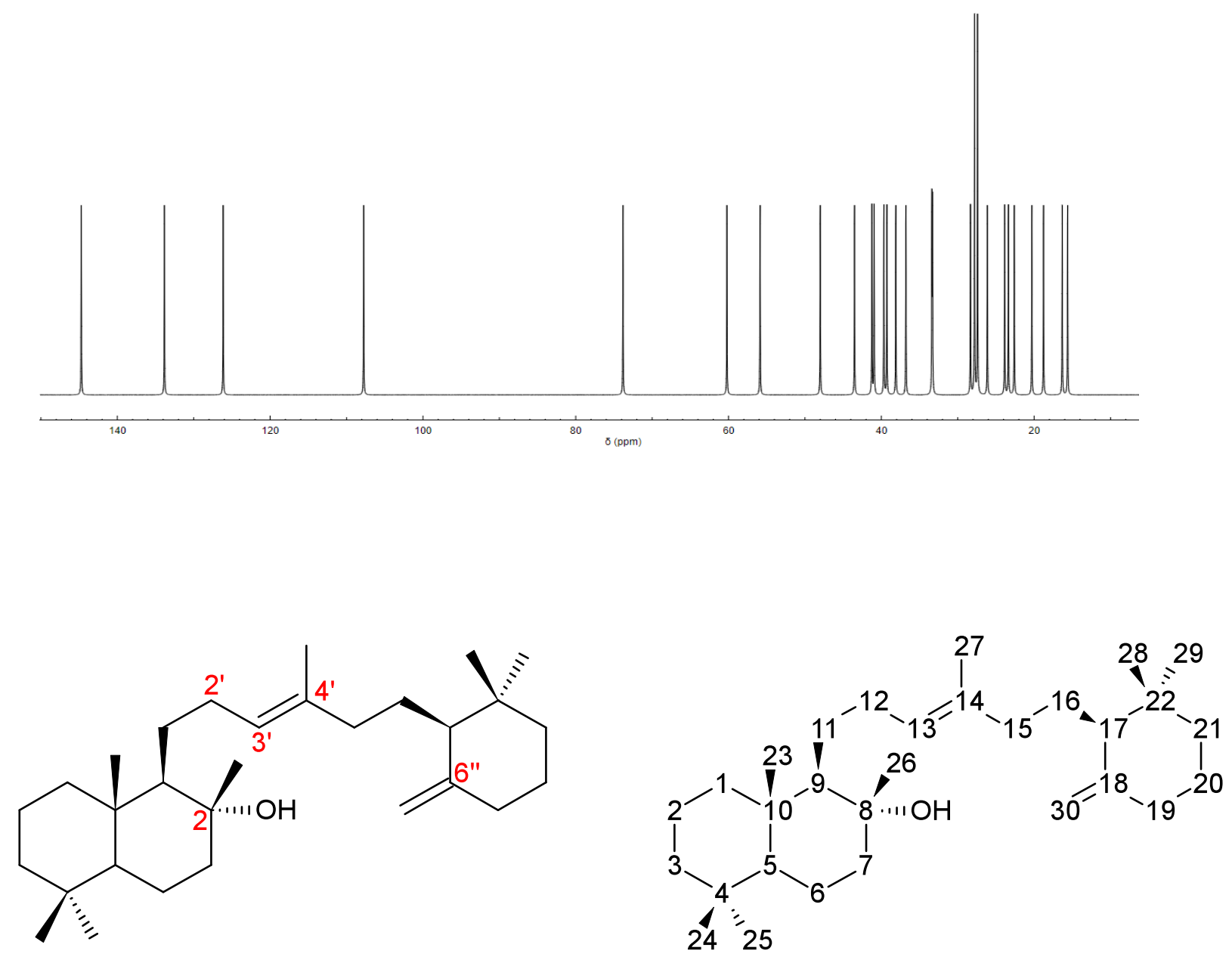
This paper is a non-peer reviewed preprint submitted to EarthArXiv.

Figure S7 DEPT NMR spectrum of fraction E from the column chromatography of ambergris extract.

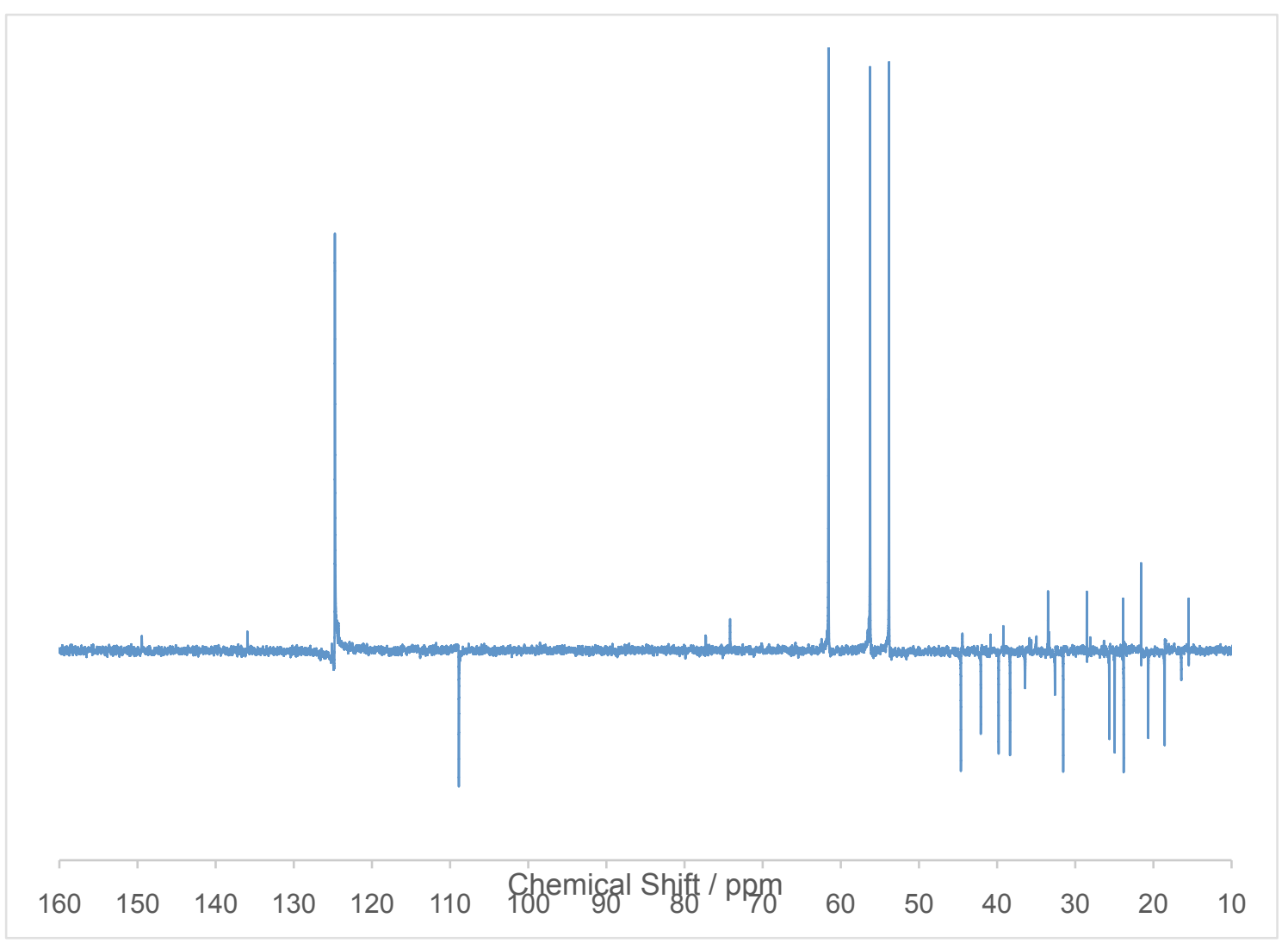

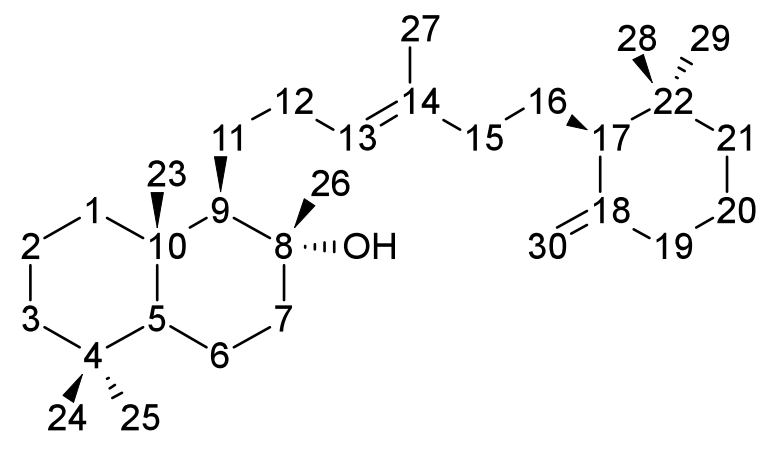


This paper is a non-peer reviewed preprint submitted to EarthArXiv.

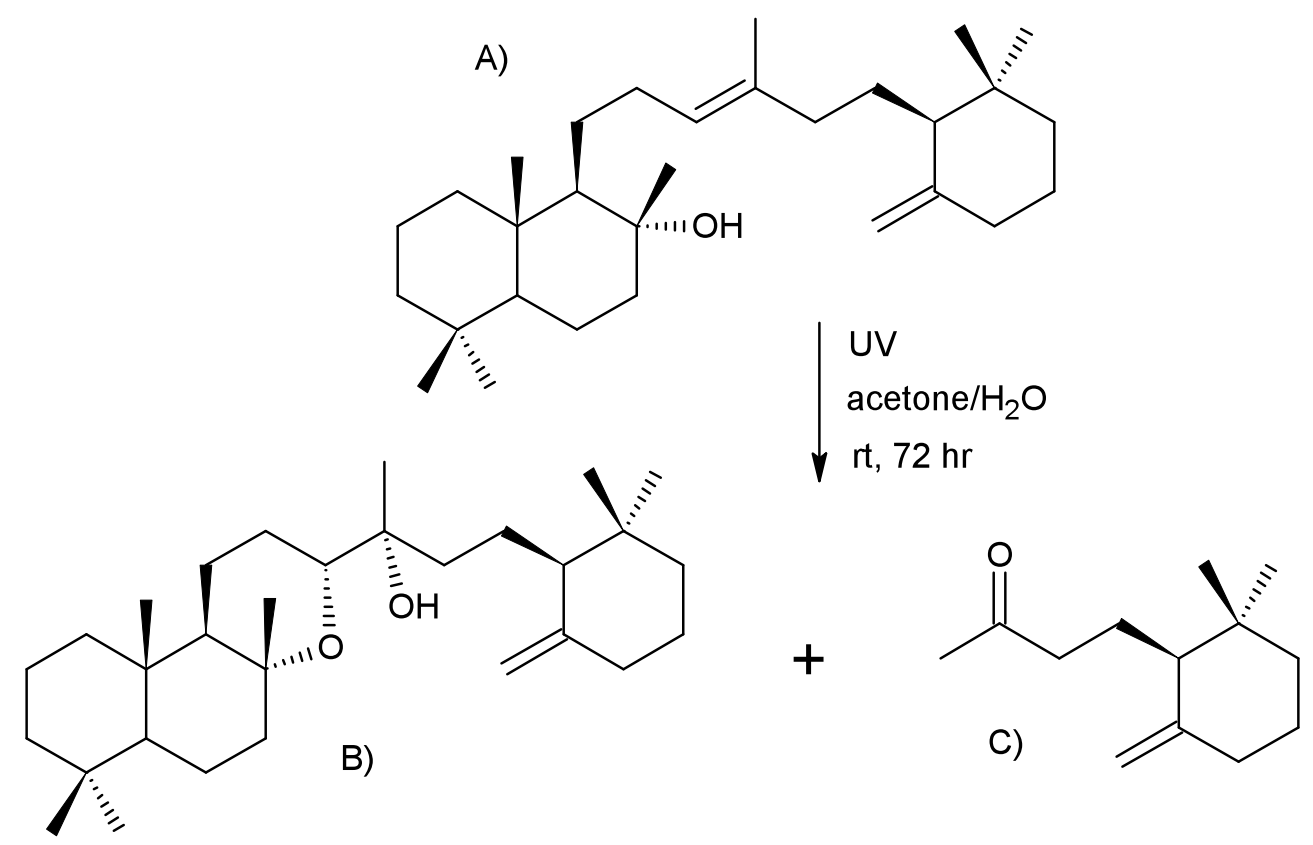

Figure S8 Intermediates in photooxidation of ambrein suggested by Shen et al. (2007).

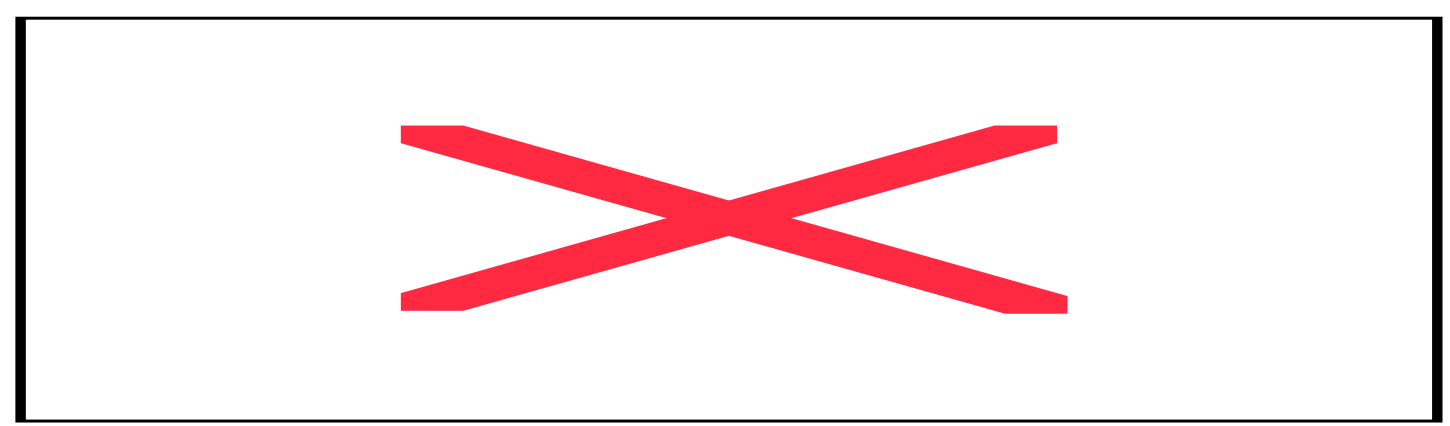

Figure S9 Intermediates in photooxidation of ambrein suggested by Ohloff et al. (1977). 